\title{
The Application of Super hydrophobic Surface Technology in the
}

\section{Corrosion Protection Field}

\author{
Yao Tao \\ North China Electric Power University in Baoding City, Hebei Province, Chna
}

Keywords: super hydrophobic, preparation, application

\begin{abstract}
In recent years, with the continuous development of science and technology, the super hydrophobic materials have wide range of application in people's daily life and various production departments. Therefore, the preparation and application of super hydrophobic materials cause the great attention of the scholars from all social circles. By reducing the free energy on the materials surface and changing the microstructure on the surface the hydrophobic property of materials can achieve the control of wet ability for the surface of solid materials so as to realize the super hydrophobicity. Based on this theory, we use a variety of technical means by controlling the reaction time and other conditions to build the materials surface of micro-nanostructure so as to make the materials obtain the hydrophobic property in the paper.
\end{abstract}

\section{Introduction}

Among the oilfield pipelines, the most severe corrosion among the corrosions is the "Three water and one heat" pipelines, namely the pipeline mixed with water, the injection pipeline, the backwater pipeline and the mixed hot pipeline. In addition, oil well pipe, casing, oil and gas pipeline corrosion are very serious too. Among the storage tanks, the wastewater tank, the high water cut oil tank, the water filling tank, the settling tank, the pressure filter tank and other tanks have the most severe corrosion among the corrosions. Because the work environments, medium conditions, varieties of materials of pipelines and storage tanks are different, the degrees of corrosion are different too. Therefore, we can't treat the corrosion reasons as the same, and we can only give some analysises on the influencing factors in the paper.

The research on the superhydrophobic surface has already had decades of development history. Even though people have had great progress in the research of this field, they have used many kinds of methods to prepare the superhydrophobic surfaces with manifold excellent performances, at present there are many problems which need to be discussed and studied. For example, the instrument for the preparation of hydrophobic materials is rather expensive, the process is very complex, and comparatively time consuming. The superhydrophobic property of the obtained materials is good. However, due to the vulnerability of rough surface, it is difficult to meet the requirements of people on the material surface properties at the same in the process of building rough surface. For this reason, we make use of the simple and effective method to build the coarse structure. Thus, obtaining the superhydrophobic surface with rather excellent property is the goal which people are constantly seeking. At the same time we can carry out mass production of the superhydrophobic materials and make them be effectively applied to each field of production and life. The preparation of practical superhydrophobic materials will become the ultimate goal of research in this field. 


\section{The Preparation Method of Superhydrophobic Materials at Home and Abroad}

Since Wenzel and Cassie have pioneered the report of the influence of surface roughness on wettability, many reports about this aspect have appeared one after another. The superhydrophobic surface in the textile, coating, lossless liquid delivery and other aspects has showed the extremely broad application prospect and has attracted people's great attentions. Especially since 1997, the German scientist Barthl Degrees. tt W. and Neinhuis C. had a systemic study on the hydrophobic property of plant leaf surface, and illuminated the critical role of the surface micro structure played in the formation of hydrophobic property, and revealed the inner mechanism of self-cleaning phenomenon on plant leaf surface. How to use the surface design and modifications of materials to make the coating be able to have the hydrophobic property like lotus leaf became the research hotspot of functional materials internationally. And this prompted the two aspects of theoretical analysis and the preparative technique to both obtain the fast development. Known from the previous discussion, the wettability of solid surface depends on its chemical composition (or surface free energy) and geometric microstructure( or surface roughness). Low surface energy material is the most basic condition of hydrophobic property, and the surface micro structure is the key factor of improving the hydrophobic property apparently. Therefore, the preparation of superhydrophobic coating always can lower the surface energy, and at the same time it is accompanied by the construction of surface micro-nano structure. Nakajima and other people have summarized people's research results in this field before this century and have divided the research process into two stages. Starting from 1950s it is the basic research stage and starting from 1990s it is the research stage of technological process and method. At present, the preparation research of superhydrophobic surface has made great achievements.

\section{The Preparation of Superhydrophobic Conversion Coating with the Solution Immersion Method}

The preparation of superhydrophobic materials is the current hot topic, and the contact angles of superhydrophobic material surface are all more than $150^{\circ}$. It has a broad application prospect in industrial and agricultural production and people's daily life. For example, if we make the hydrophobic materials be applied in the ship outer shell, and the fuel storage tank, it can achieve the results of preventing the corrosion, reducing the friction, preventing the pollution and others. If we make the hydrophobic materials be used in the petroleum pipeline transportation, it can prevent the adhesion of petroleum on the pipeline wall so as to reduce the loss and the energy consumption in the process of transportation and reduce the congestion problem of petroleum pipeline. We can also make the hydrophobic materials be applied in the textile, make the waterproofing and antifouling clothes and others. Because the superhydrophobic materials have broad research value and application prospect, people make use of a variety of ways and means to prepare this kind of materials. People use the electrochemistry to corrode the metals so as to prepare the superhydrophobic surface. People use the method of vapor deposition to prepare the superhydrophobic surface. People use the method of phase separation to prepare a kind of superhydrophobic surface. Tuteja and other people reported several methods of preparing the super double hydrophobic materials and others on Science in 2007. These research results mentioned above have very important significance and are expected to appear in the practical application for the theoretical research of the super hydrophobic surface. Even so, there are some problems which still need to be solved, such as the strict preparatory condition, the laborious procedures, the expensive materials and rather poor stability and durability. Therefore, using cheap materials, 
looking for a simple and feasible method, and preparing the superhydrophobic surface with the stable environment have been becoming the current major issue.

As is known to all, among a variety of chemical methods, the solution immersion method is a kind of the simplest preparation method. In this chapter, we use this kind of method to prepare the superhydrophobic materials. That is to say, we build the superhydrophobic conversion coating with the stable environment on the metal surface. The characteristics of the method is simple, cheap and it can be prepared in large area. Thus, the superhydrophobic surface has the very good environmental stability. The surface topography and composition can be represented by the scanning electron microscope and the X-ray photoelectron spectroscopy. The surface wettability of materials can be represented by the contact angle.

\section{The Experiment Part.}

(1) The Experimental Materials and Instruments

The experimental drugs: Octadecylamine, dicyclohexylaminenitrite, cyclohexanone solution, dilute nitric acid(5 \%), the second deionized water, and absolute ethyl alcohol. The above drugs are all chemically pure drugs.

The experimental test block: Q235 steel (The specification is $50 \mathrm{~mm} \times 25 \mathrm{~mm} \times 2 \mathrm{~mm}$ ).

The experimental instruments: Beaker, electronic balance, glass rod, measuring cylinder, digital camera, scanning electron microscope(Hitachi S-3400N), energy disperse spectroscopy(Oxford INCA - 350) and contact angle tester(JY-82).

(2) The Experimental Procedure

The Configuration of Cleaning Steel Disc and Hydrophobic Reagent

(a)We make the steel disc be polished by using the sandpaper to remove the pollutants and the oxidation layer.

(b)We add the octadecylamine with the weight of $1 \mathrm{~g}$ and the dicyclohexylaminenitrite with the weight of $1 \mathrm{~g}$ into the cyclohexanone solution with the weight of $40 \mathrm{~g}$, then heat with frequent agitation and boil to completely dissolve the solution so as to produce the superhydrophobic reagent.

The Configuration of Superhydrophobic Conversion Coating

We make the steel disc be immersed in the dilute nitric acid with $5 \%$ concentration. After $90 \mathrm{~min}$, we take the steel disc out of the dilute nitric acid with $5 \%$ concentration. After the repeated washing of the second deionized water and ethanol, we make the steel disc be immersed in the hydrophobic reagent for $24 \mathrm{~h}$ so as to conduct the second surface treatment. After that we use the absolute ethyl alcohol to wash and then blow the steel disc dry.

The Property Characterization of Superhydrophobic Conversion Coating

(a)We make use of Hitachi S-3400N scanning electron microscope to observe the super hydrophobic surface topography.

(b)We measure the contact angle by using the deionized water, and the liquid volume is $5 \mu \mathrm{L}$. We use JY-82 contact angle measuring instrument to measure the static contact angle of water on the superhydrophobic surface.

The Experimental Conclusion. We use the dropper to drop the little drops of water on the test block a and b respectively to observe its appearance shape. The test block a is the original steel disc without any soak and second processing. When the little drops of water are dropped on the original steel disc, we can see that the little drops of water present the state of hemisphere on its surface. The little drops of water are very stable the original steel surface and they are not likely to slide. At this moment the contact angle is only 80 degrees. When the rolling Angle reaches 20 degrees or so, the steel surface is in the water loving state. After the test block b goes through the dilute nitric acid's etching for $90 \mathrm{~min}$ and then it is immersed in the hydrophobic reagent, so we obtain the 
superhydrophobic surface. When the little drops of water can't stay stable on the superhydrophobic surface and they are likely to slide, at this moment the contact angle of conversion coating surface reaches 140 degrees and the rolling Angle reaches 4 degrees.

Different redox response time has the influence on the dimension of hydrophobic property of superhydrophobic conversion coating surface. As far as the steel disc is concerned, its contact angle increases gradually with the extension of response time, and the contact angle can be increased from the initial 80 degrees to 140 degrees after $90 \mathrm{~min}$. When we make the steel disc be polished by using the sandpaper to remove the oxidation layer and the pollutants, the steel disc surface is smooth basically and it doesn't change obviously. When the etching time reaches about 30 min, we take the steel disc out. After it is rinsed by using a large number of ionized water and ethanol, we can observe the structure of steel disc surface under the scanning electron microscopy, and we can see the nano-scale particles with the bumpy and uneven distribution on the steel disc surface. At this moment the contact angle of steel disc surface can reach 100 degrees. The response continues to proceed. When the time is extended to $90 \mathrm{~min}$, we can use the scanning electron microscopy to observe again, and we can see that a large number of nano-scale particles with the uniform distribution and the similar shape are formed on the steel disc surface. The little drops of water are not stable on its surface, and the contact angle reaches 120 degrees.

\section{Conclusion}

We adopt the solution immersion method to prepare the superhydrophobic metal conversion coating. When the steel disc reacts with dilute nitric acid, the steel disc surface is etched, and the rough structure with ups and downs is formed on its surface. This kind of rough surface structure is more advantageous to the formation of superhydrophobic conversion coating. At the same time we also investigate various factors which influence the rough structure of superhydrophobic conversion coating surface, and the time length of conducting the redox response has a direct effect on the superhydrophobic property of metal surface. When the response begins to proceed, the rough structure on the metal surface is not obvious. However, the rough structure on the metal surface will become apparent with the extension of response time. The hydrophobic reagent also plays a crucial role for the conversion coating. For the same metal materials, before they are immersed and after immersed in the hydrophobic reagent the contact angles on the metal surface have changed a lot. At the same time when we conduct the experiments of the superhydrophobic metal conversion coating in various complicated environments, good corrosion resistance and the superhydrophobic property both be presented on the conversion coating surfaces.

\section{References}

[1]Chen Ruijiao. The Preparation of Superhydrophobic Surface with the Electrophoresis Method and Its Applications in Metal Protection[D]. Zhejiang University, 2017.

[2]Liu Jiayue.The Research on the Preparation of Superhydrophobic Polyaniline and Its Corrosion Resistance Property[D]. Lanzhou University of Technology, 2016.

[3]Zhu Wenzhong. The Research on the Preparation and Endurance Quality of Elastic SiO_2 / Polymer Superhydrophobic Polymer Materials[D]. Henan University, 2016.

[4]Zhao Wei.The Electrochemical Preparation of Nano-micro Structure Superhydrophobic Surface on the Metallic Copper Base[D]. Hunan Normal University, 2016.

[5]Chen Jiaqi. The Preparation and Property of Aluminium Base Super Double Hydrophobic Materials[D]. South China University of Technology, 2016. 
